\title{
EFECTO ANTIANÉMICO DE LAS HOJAS DE REMOLACHA EN GESTANTES PRIMÍPARAS QUE ACUDIERON AL CAP II MACACONA-ESSALUD DE ICA. 2019
}

\author{
Anti -anemic effect of beet leaves in pregnant primiparous who attended the CAP II Macacona- \\ EsSalud of Ica. 2019
}

Mariela Rosario Zegarra Zeballos ${ }^{1 c d}$, Héctor William Carlos Cruces ${ }^{2, a, e}$, Belinda Marleni Navarro Guerra ${ }^{2 f}$, Thalía Jesalem Carlos Campos ${ }^{1 b}$

\footnotetext{
${ }^{1}$ Hospital Augusto Hernández Mendoza Es Salud-Ica, Perú

${ }^{2}$ Docente de la Facultad de Ciencias Económicas y Negocios Internacionales. Universidad Nacional San Luis Gonzaga. Ica, Perú

${ }^{a}$ Economista. ${ }^{b}$ Licenciada en Enfermería, ${ }^{C}$ Obstetra, ${ }^{d}$ Doctor en Salud Pública, ${ }^{e}$ Doctor en Educación, ${ }^{f}$ Doctor en Ciencias Administrativas
}

\section{RESUMEN}

En Perú, según OMS, la anemia es un problema severo de salud pública que afecta al $42 \%$ de madres gestantes. Prevalencia de anemia en Ica $18.3 \%$ siendo los promedios nacionales $28 \%$ (ENDES 2015). EI CAP II Macacona-EsSalud, en 2018 tiene MEF de 7520, 104 anemia gestacional. Porcentaje alto evidenciaron intolerancia al sulfato ferroso. De las 55 mujeres con $\mathrm{Hb}$ $11 \mathrm{~g} / \mathrm{dL}$ fueron seleccionadas 40 mujeres. Dividiéndolas en 2 grupos: Grupo A consumo de Sulfato ferroso y ácido fólico, Grupo B consumo $300 \mathrm{gr}$ día de remolacha cruda ya que con la cocción se pierden propiedades. Ambos por 3 meses, se tomó criterios de inclusión en ambos grupos: edad de 20 - 36 años, gestantes primer y segundo trimestre, Primíparas, Hemoglobina $11 \mathrm{~g} / 100 \mathrm{~mL}$, sin patología. Objetivo: comprobar si, el efecto antianémico del consumo del sulfato ferroso y ácido fólico es el mismo al de las hojas de Beta vulgaris (Remolacha). Material y Métodos: Estudio experimental, prospectivo, de corte longitudinal, analítico de nivel aplicativo. Resultados: Se aplicó prueba Fisher, Correlación lineal, t de Student. Trascurrido tres meses se realiza un segundo dosaje de Hb.: Grupo A Hb 11,71 g/dL, Grupo B 12,61 g/dL, indicando en el Grupo A Hb aumenta $0,71 \mathrm{~g} / \mathrm{dL}$ y $1,61 \mathrm{~g} / \mathrm{dL}$ en el Grupo B. Conclusiones: El consumo de remolacha aporta hierro y ácido fólico.

Palabras clave: Remolacha, Anemia, Sulfato ferroso, ácido fólico.

\section{SUMMARY}

In Peru, according to $\mathrm{WHO}$, anemia is a severe public health problem that affects $42 \%$ of pregnant mothers. Prevalence of anemia in Ica, city of Peru, 18,3\%, with national averages $28 \%$ (ENDES 2015). The "CAP II Macacona-EsSalud" medical center, in 2018 has MEF of 7520, 104 gestational anemia. High percentage showed intolerance to ferrous sulfate. Of the 55 women with $\mathrm{Hb} 11 \mathrm{~g} /$ $\mathrm{dL}, 40$ women were selected. Dividing them into 2 groups: Group A consumption of ferrous sulfate and folic acid, Group B consumption 300-gr day of raw beet since with cooking properties are lost. Both for 3 months, inclusion criteria were taken in both groups: age 20 - 36 years, first and second trimester pregnant women, Primiparas, Hemoglobin $11 \mathrm{~g} / 100 \mathrm{~mL}$, no pathology. Objective: to verify if, the antianemic effect of the ferrous sulfate and folic acid consumption is the same as that ofthe leaves of Beta vulgaris (Beet). Materials and Methods: Experimental and prospective study, of longitudinal, analytical and application level. Results: "Fisher" was applied. "Linear correlation", "Student's t". After three months, a second dose of $\mathrm{Hb}$ was carried out.: Group A Hb 11,71g / dL, Group B 12,61g / dL, indicating in Group A Hb increases 0,71g / dL and 1,61g / dL in Group B. Conclusions: Consumption of beet contributes iron and folic acid.

Key words: Beet, Anemia, Ferrous sulfate and folic acid. 


\section{INTRODUCCIÓN}

La anemia materna es considerada un importante problema de salud pública. Las tasas más altas se encuentran en los países más pobres, particularmente en África, donde en la región occidental y central tienen una tasa de 40 y $50 \%$ para mujeres no embarazadas $\quad y$ embarazadas, respectivamente. Se asume que la deficiencia de hierro es la principal causa de esta alta tasa de anemia. En África, por ejemplo, se asocia fuertemente la deficiencia de hierro con los niveles socioeconómicos más bajos (1). En países en desarrollo la prevalencia de la anemia promedio es del $56 \%$ con valores entre el 35 y $75 \%$ entre diferentes regiones del mundo. Situación opuesta a la de países desarrollados que tienen una prevalencia promedio de $18 \%$. América Latina tiene $39 \%$ de mujeres gestantes con niveles bajos de hemoglobina, que se distribuyen en $37 \%$ en América Central y del Sur y $52 \%$ en el Caribe (2).

La anemia es la alteración hematológica que más se diagnostica durante el embarazo, producida básicamente por los cambios en el volumen corporal total materno al expandirse para lograr la adecuada perfusión feto placentaria y prepararse para amortiguar las pérdidas durante el parto (3). En la mujer embarazada se determina por una concentración de hemoglobina $(\mathrm{Hb})$ menor de $11 \mathrm{~g} / \mathrm{dl}$, conforme a los criterios de la Organización Mundial de la Salud (OMS) (4). La anemia materna se clasifica en grados de acuerdo al nivel de hemoglobina. Así se tiene la anemia leve $(\mathrm{Hb}<11-9 \mathrm{~g} / \mathrm{dL})$, la anemia moderada $(\mathrm{Hb}<9-7 \mathrm{~g} / \mathrm{dL})$ y la anemia severa $(\mathrm{Hb}<7 \mathrm{~g} / \mathrm{dL})$ (5). En Perú, en el año 2011, la tasa de anemia materna en las mujeres gestantes atendidas en hospitales del sector público fue $18,1 \%$. Según la severidad de la anemia, se halló un $16,6 \%$ de casos de anemia leve, un $1,4 \%$ de anemia moderada y un $0,1 \%$ de anemia severa (6).
El suplemento de hierro durante el embarazo es un tema controvertido que involucra muchos aspectos de relevancia para lograr un resultado favorable, tanto en la madre como en el feto. Los valores normales de la hemoglobina $(\mathrm{Hb})$ y el resto de los parámetros sanguíneos han de relacionarse con el estilo de vida, la nutrición y otros factores tanto sociales como económicos, culturales y geográficos. El estilo de vida influye en la salud y puede modificar los valores normales de hemoglobina (7).

En Perú, se estima que la incidencia de mujeres gestantes que no cumplen el tratamiento con sulfato ferroso es cercana al 20\% (8). Según ENDES 2015 en el Perú hay un $28 \%$ de anemia en gestantes. La prevalencia de la anemia a nivel de la región Ica en el 2017 es de 356 casos (10,7\%) (SIEN 2017) El rechazo a la ingesta de sulfato ferroso puede verse afectada por factores como los bajos ingresos económicos, las creencias populares, el difícil acceso al establecimiento de salud (9), los estudios incompletos, el tiempo prolongado de tratamiento, la presencia de sintomatología como mareos, náuseas, vómitos y dolor abdominal y la nuliparidad (10).

Ante esta situación, el presente estudio pretende estudiar $\mathrm{Si}$ el consumo de las hojas de Beta vulgaris (Remolacha) tiene un efecto antiemético en las gestantes atendidas en el CAP II Macacona EsSalud Ica., toda vez que la ingestión de este mineral, se hace también necesario ante la presencia de anemias, leucemia o transfusiones muy habituales, posee propiedades rejuvenecedoras, cuyo consumo puede mantener la juventud durante más tiempo, también interviene en la síntesis del aminoácido metionina, cuya existencia es necesaria para la buena salud del cabello, las uñas o la piel, participa en la producción de la hormona dopamina, que nos previene del malhumor y de los síntomas depresivos, hay que destacar su riqueza en fibras muy útil para vaciar el intestino y prevenir el estreñimiento. 
es un alimento muy adecuado para los que sufran retención de líquidos, por lo que deberán comerlo habitualmente los obesos, artríticos o quienes pretendan rebajar peso., no solamente depura los riñones, sino también la sangre al resultar alcalinizante: eliminando la acidez corporal y ayudando al hígado en su función depurativo, hecho que lo hace muy interesante para que sea consumido por enfermos de hígado., estimula el cerebro y elimina las toxinas que en él se puedan acumular, por lo que ayuda a mantener una buena salud mental y prevenir el envejecimiento precoz.

EI CAP II Macacona-EsSalud, se encuentra en el distrito de Subtanjalla, departamento de Ica; cuya población adscrita incluye también. A los distritos colindantes de Guadalupe y San Juan Bautista, teniendo así en el 2018 MEF de 7520 y 104 casos de anemia gestacional, evidenciando en un gran porcentaje, intolerancia a la ingesta del sulfato ferroso; lo cual acentuó el interés por tratar de mejorar la situación. Dentro de las limitaciones del estudio se detectó que, en el monitoreo a las gestantes vía telefónica, no se aseguraba que las gestantes ingerían el producto en la hora sugerida.

\section{MATERIAL Y MÉTODOS}

Según la intervención del investigador el estudio es experimental, prospectiva, de corte longitudinal puesto que se midió la variable más de una vez y analítica por el número de variables. De nivel aplicativo. Es una investigación con diseño experimental verdadero, ya que se realizó con la manipulación deliberada de variables, y además cumple con la asignación aleatoria (grupo control) e intervención a propósito de la investigación, en su modalidad de Aplicativo, longitudinal. Se utilizó los siguientes métodos teóricos como camino a cumplir los objetivos de la investigación: El método deductivo, que determino el problema, inductivo, que estudio el problema en forma global, analítico, que analizo los resultados, sintético, que formulo conclusiones y recomendaciones, bibliográfico, que extrajo la información en textos e Internet.

Se aplicó el análisis de varianza simple Se elige la Prueba de Fisher (F). Nivel de confianza: $95 \%$, Nivel de significación: $\alpha=0.05$; correlación lineal y prueba de consistencia se elige la Prueba t de Student con un Coeficiente de correlación $(r=0,97)$. Se tuvo 55 gestantes que acudieron al CAP II Macacona-EsSalud, de la ciudad de lca, del 1 de octubre al 31 de diciembre del año 2019 a las cuales se les aplico la formula y se obtuvo 40 gestantes las cuales cumplieron todos los criterios de inclusión sobre todo el de tener $\mathrm{Hb}$ de $11 \mathrm{~g} / \mathrm{dl}$.

- Edad de 20 - 36 años

- Gestantes del primero y segundo trimestre Primíparas

- Nivel de Hemoglobina de $11 \mathrm{~g} / 100 \mathrm{~mL}$

- Ausencia de patología

Se las dividió en dos grupos de 20 gestantes; el grupo A recibió $60 \mathrm{mg}$., de sulfato ferroso y 250 ug de ácido fólico diario y el grupo B de 20 gestantes también que se les recomendará el consumo de hojas de remolacha de $300 \mathrm{~g}$. de hojas de Beta Vulgaris (Remolacha). Los instrumentos de recolección de datos fueron la Encuesta y la historia clínica. El instrumento fue elaborado por la autora de la investigación y validado mediante juicio de cinco profesionales expertos en el área de salud. Cabe indicar que la investigación previa a su ejecución tuvo el respectivo tramite y posterior autorización del comité de ética de la Red Asistencial Ica de EsSalud, el cual remitió una Resolución formalizando el hecho (Carta №107-DHIV-AHM-GRA-ICA-ESSALUD-2018. Aprobación proyecto de investigación y carta №3-Comité de ética en investigación). Se aplicó tablas dinámicas en Microsoft Excel para la determinación de los niveles de hemoglobina y las prevalencias respectivas. Se empleó el programa SPSS versión 23 para el análisis estadístico de los datos, 
empleándose el análisis de varianza simple Se elige la Prueba de Fisher (F). Nivel de confianza: $95 \%$, Nivel de significación: $\alpha=0.05$; correlación lineal y prueba de consistencia se elige la Prueba t de Student con un Coeficiente de correlación $(r=0,97)$.

\section{RESULTADOS}

Se las dividió en dos grupos de 20 gestantes; el grupo A recibió $60 \mathrm{mg}$., de sulfato ferroso y 250 ug de ácido fólico diario y el grupo B de 20 gestantes también que se les recomendará el consumo de hojas de remolacha de $300 \mathrm{~g}$. de hojas de Beta Vulgaris (Remolacha). Después de tres meses se le realiza un dosaje de $\mathrm{Hb}$ cuyos resultados se enuncian en la Tabla 1.

Una vez trascurrido los tres meses se les realiza un segundo dosaje de $\mathrm{Hb}$. (Tabla II), obteniendo en el Grupo A un promedio de $\mathrm{Hb}$ de $11,71 \mathrm{~g} / \mathrm{dLy}$ en Grupo B de $12,61 \mathrm{~g} / \mathrm{dL}$ (Cuadro II) eso indica que en promedio se aumentó en un $0,71 \mathrm{~g} / \mathrm{dL}$ en el Grupo $A$ y $1,61 \mathrm{~g} / \mathrm{dL}$ en el Grupo B, apreciado en la Tabla 2.

Según la contrastación de la hipótesis estadística aplicando la Varianza simple, se rechaza la hipótesis nula con un nivel de significancia del 95\%, por lo tanto, la influencia de los tratamientos no sería igual. Teniendo en cuenta los resultados es evidente la influencia positiva del consumo de remolacha como un agente antianémico, por el aporte de hierro y ácido fólico. Al probar la hipótesis para establecer si existe o no correlación entre los datos, se llegó a rechazar la hipótesis nula de la nutrición en gestantes en pamplona alta, red SJM - VMT. DISA II Lima sur 2006. En el cual concluyen que Los alimentos ricos en hierro no son de su agrado, Los alimentos proteicos son poco consumidos. Lo cual fortalece la inquietud que la investigación quiso demostrar con el uso de algún apoyo (hojas de remolacha) en beneficio de la nutrición en gestantes con baja hemoglobina. (12).
Tabla 1.

Hemoglobina (g/dl) en gestantes. grupo a (consumo de sulfato ferroso y ácido fólico), grupo b (consumo de hojas de remolacha) después de tres meses

\begin{tabular}{ccc}
\hline Caso & $\begin{array}{c}\text { Sulfato ferroso } \\
\text { yácido fólico } \\
\text { A }\end{array}$ & $\begin{array}{c}\text { Hojas de } \\
\text { remolacha } \\
\text { B }\end{array}$ \\
1 & 11.40 & 12.30 \\
2 & 12.80 & 13.10 \\
3 & 12.00 & 12.80 \\
4 & 11.20 & 12.60 \\
5 & 12.70 & 12.60 \\
6 & 11.00 & 12.30 \\
7 & 12.40 & 12.80 \\
8 & 11.30 & 12.20 \\
9 & 11.30 & 12.60 \\
10 & 11.00 & 12.40 \\
11 & 12.20 & 13.00 \\
12 & 11.60 & 12.60 \\
13 & 11.40 & 12.30 \\
14 & 12.80 & 13.60 \\
15 & 11.50 & 12.80 \\
16 & 11.00 & 11.80 \\
17 & 11.60 & 12.80 \\
18 & 12.60 & 13.20 \\
19 & 11.00 & 12.00 \\
20 & 11.30 & 12.30 \\
\hline
\end{tabular}

Tabla 2. Promedios de hemoglobina obtenidos en el grupo A y grupo B de gestantes

\begin{tabular}{ccc} 
Grupo A & Grupo B & Total \\
234.10 & 252.10 & 486,20 \\
20 & 20 & 40 \\
11.705 & 12.605 & 12.155 \\
$\mu 1$ & $\mu 2$ & \\
\hline
\end{tabular}

\section{CONCLUSIONES}

Según la contrastación de la hipótesis estadística aplicando la Varianza simple, se rechaza la hipótesis nula con un nivel de significancia del $95 \%$, por lo tanto, la influencia de los tratamientos no sería igual. Teniendo en cuenta los resultados es evidente la influencia positiva del consumo de remolacha como un agente antianémico, por el aporte de hierro.

Al probar la hipótesis para establecer si existe o no correlación entre los datos, se llegó a rechazar la hipótesis nula de la Prueba de consistencia, pues es evidente que ha habido incremento en los niveles de hemoglobina para 
ambos tratamientos, lo cual se refleja con el Coeficiente de correlación positivo de 0,97

\section{RECOMENDACIONES}

En principio se debe sensibilizar a las mujeres acerca de la anemia, a través de capacitaciones en su proceso de espera de consulta, en los establecimientos de salud, no solamente en etapa de gestación, más al contrario debería convertirse en una transversal para el cuidado de las personas.

Se debe coadyuvar con las Redes Asistenciales de EsSalud, u otras instituciones a fin de generar charlas educativas, en ferias de salud, colegios y todo aquel evento que permita la inclusión de este tema, es decir, proyectándolo como una transversal por la importancia que representa. Dado los resultados del estudio nos atrevemos a sugerir el consumo de este producto natural, que aparte de ser asequible y barato es muy bien tolerado en el ser humano.

Promover la inclusión de este vegetal en la dieta, por sus variados beneficios, no sólo limitado al aporte de hierro, y otros como ácido fólico, síntesis del aminoácido metionina, dopamina, silicio, potasio, fosforo, magnesio, zinc, calcio, sodio etc, siendo muy interesante para su consumo en las mujeres, quienes necesitan fundamentalmente estos elementos durante el embarazo, reforzando a mantener una buena salud física y mental.

\section{CORRESPONDENCIA}

Obsta. Dra. Mariela Rosario Zegarra Zeballos Correo electrónico: maryde2568@yahoo.com

\section{REFERENCIAS BIBLIOGRÁFICAS}

1. Ayoya $\mathbf{M}$, Bendech $\mathbf{M}$, Zagré $\mathbf{N}$, Tchibindat F. Maternal anaemia in West and Central Africa: time for urgent action. Public Health Nutrition. 2012; 15:916-27.

2. Rasmussen, KM. Is there a causal relationship between iron deficiency or iron deficiency anemia and weight at birth, length of gestation and perinatal mortality? J Nutr 131, 2 Suppl. 2, 590S-603S.Google Scholar PubMed. 2001.

3. Hill C, Pickinpaugh J. Physiologic changes in pregnancy. The Surgical clinics of North America. 2008; 88(2):391-401.

\section{World Health Organization \& UNICEF} Focusing on Anaemia: Towards an Integrated Approach for Effective Anaemia Control. Geneva: WHO; available at http://www.who.int/nutrition/publications/W HOandUNICEF_statement_anaemia.pdfG oogle Scholar; 2004.

5. Montoya R, Castelazo M, Valerio C. Opinión de un grupo de expertos en diagnóstico y tratamiento de la anemia en mujer embarazada. GinecolObstetMex. 2012; 80:563-80.

6. Gonzales G, Tapia V, Gasco M, Carrillo C. Hemoglobina materna en el Perú: diferencias regionales y su asociación con resultados adversos perinatales. Rev Peru Med ExpSaludPublica 2011; 28(3):484-91.

7. Cogswell, M, Parvanta, I, Ickes, L. Iron supplementation during pregnancy, anemia, and birth weight: a randomized controlled trial. Am J Clin Nutr 78, 773781.Google ScholarPubMed. 2003

8. Ramos Y. Factores asociados a la suplementación de sulfato ferroso en gestantes anémicas en el Hospital de Rioja, 2016. Huánuco: Universidad de Huánuco; 2017.

9. Espinoza K. Factores que influyen en la adherencia al sulfato ferroso como tratamiento y prevención de la anemia en gestantes del Hospital Apoyo Puquio 2015. Ica, Perú: Universidad Alas Peruanas; 2015.

Recibido: 18/04/2021

Aprobado para publicación: 23/08/2021 